\title{
Dark Matter Superfluidity
}

\author{
Justin Khoury* \\ Center for Particle Cosmology, Department of Physics and Astronomy, University of \\ Pennsylvania, Philadelphia, PA 19104 \\ jkhouryesas . upenn. edu
}

In this talk I summarize a novel framework that unifies the stunning success of MOND on galactic scales with the triumph of the $\Lambda$ CDM model on cosmological scales. This is achieved through the rich and well-studied physics of superfluidity. The dark matter and MOND components have a common origin, representing different phases of a single underlying substance. In galaxies, dark matter thermalizes and condenses to form a superfluid phase. The superfluid phonons couple to baryonic matter particles and mediate a MOND-like force. This framework naturally distinguishes between galaxies (where MOND is successful) and galaxy clusters (where MOND is not): dark matter has a higher temperature in clusters, and hence is in a mixture of superfluid and normal phase. The rich and well-studied physics of superfluidity leads to a number of striking observational signatures, which we briefly discuss. Remarkably the critical temperature and equation of state of the dark matter superfluid are similar to those of known cold atom systems. Identifying a precise cold atom analogue would give important insights on the microphysical interactions underlying DM superfluidity. Tantalizingly, it might open the possibility of simulating the properties and dynamics of galaxies in laboratory experiments.

The 11th International Workshop Dark Side of the Universe 2015

14-18 December 2015

Kyoto, Japan

${ }^{*}$ Speaker. 


\section{Introduction}

In the $\Lambda$-Cold-Dark-Matter $(\Lambda \mathrm{CDM})$ standard model of cosmology, dark matter (DM) consists of collisionless particles. This model does exquisitely well at fitting a number of large-scale observations, from the background expansion history to the cosmic microwave background anisotropies to the linear growth of cosmic structures [1].

On the scales of galaxies, however, the situation is murkier. A number of challenges have emerged for the standard $\Lambda \mathrm{CDM}$ model in recent years, as observations and numerical simulations of galaxies have improved in tandem. For starters, galaxies in our universe are surprisingly regular, exhibiting striking correlations among their physical properties. For instance, disc galaxies display a remarkably tight correlation between the total baryonic mass (stellar + gas) and the asymptotic rotational velocity, $M_{\mathrm{b}} \sim v_{\mathrm{c}}^{4}$. This scaling relation, known as the Baryonic Tully-Fisher Relation (BTFR) [2, 3], is unexplained in the standard model. In order to reproduce the BTFR on average, simulations must finely adjust many parameters that model complex baryonic processes. Given the stochastic nature of these processes, the predicted scatter around the BTFR is much larger than the observed tight correlation [4]. Simulated dwarf galaxies fail to account for the diversity of observed rotation curves [5].

Another suite of puzzles comes from the distribution of dwarf satellite galaxies around the Milky Way (MW) and Andromeda galaxies. The $\Lambda$ CDM model predicts hundreds of small DM halos orbiting MW-like galaxies, which are in principle good homes for dwarf galaxies, yet only $\sim 20-30$ dwarfs are observed around the MW and Andromeda. Recent attempts at matching the populations of simulated subhaloes and observed MW dwarf galaxies have revealed a "too big to fail" problem [6]: the most massive dark halos seen in the simulations are too dense to host the brightest MW satellites. Even more puzzling is the fact that the majority of the MW [7] and Andromeda $[8,9]$ satellites lie within vast planar structures and are co-rotating within these planes. This suggests that dwarf satellites did not form independently, as predicted by the standard model, but may have been created through an entirely different mechanism [7, 10].

A radical alternative is MOdified Newtonian Dynamics (MOND) [11, 12]. MOND replaces DM with a modification to Newton's gravitational force law that kicks in whenever the acceleration drops below a critical value $a_{0}$. For large acceleration, $a \gg a_{0}$, the force law recovers Newtonian gravity: $a \simeq a_{\mathrm{N}}$. At low acceleration, $a \ll a_{0}$, the force law is modified: $a \simeq \sqrt{a_{\mathrm{N}} a_{0}}$. This simple empirical law has been remarkably successful at explaining a wide range of galactic phenomena [13]. In particular, asymptotically flat rotation curves and the BTFR are exact consequences of the force law. ${ }^{1}$ MOND does exquisitely well at fitting detailed galactic rotation curves, as shown in Fig. 1. There is a single parameter, the critical acceleration $a_{0}$, whose best-fit value is intriguingly of order the speed of light $c$ times the Hubble constant $H_{0}: a_{0} \simeq \frac{1}{6} c H_{0} \simeq 1.2 \times 10^{-8} \mathrm{~cm} / \mathrm{s}^{2}$.

However, the empirical success of MOND is limited to galaxies. The predicted X-ray temperature profile in massive clusters of galaxies is far from the observed approximately isothermal profile [18]. Relativistic extensions of MOND, e.g. [19], fail to reproduce CMB anisotropies and

\footnotetext{
${ }^{1}$ Consider a test particle orbiting a galaxy of mass $M_{\mathrm{b}}$, in the low acceleration regime. Equating the centripetal acceleration $v^{2} / r$ to the MONDian acceleration $\sqrt{a_{\mathrm{N}} a_{0}}=\sqrt{\frac{G_{\mathrm{N}} M_{\mathrm{b}} a_{0}}{r^{2}}}$, we obtain a velocity that is independent of distance, $v^{2}=\sqrt{G_{\mathrm{N}} M_{\mathrm{b}} a_{0}}$, in agreement with the flat rotation curves of spiral galaxies. Squaring this gives the BTFR relation $M_{\mathrm{b}}=\frac{v^{4}}{G_{\mathrm{N}} a_{0}}$ as an exact prediction.
} 


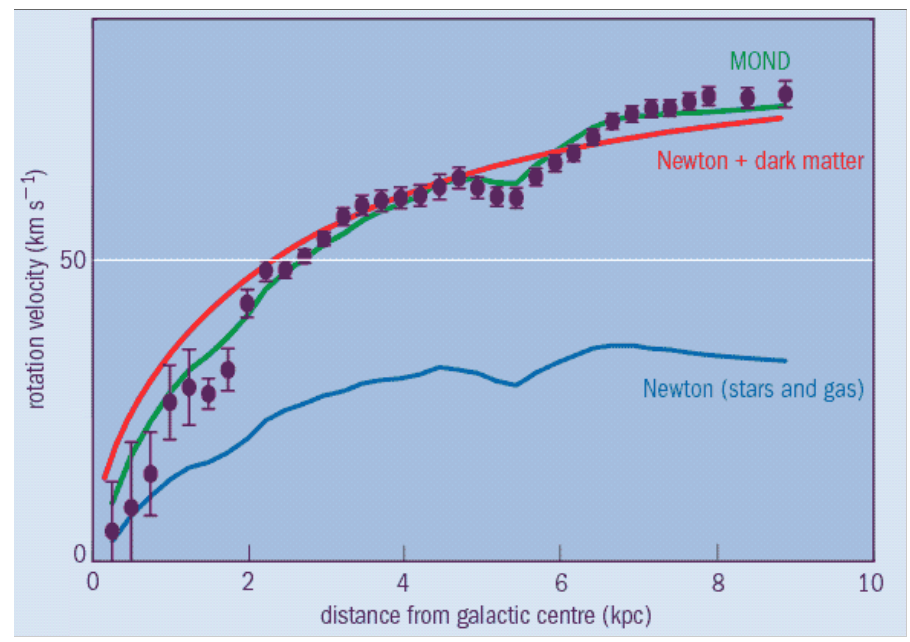

Figure 1: Observed rotation curve for NGC1560 (blue points) [14]. The MOND curve (green) [15] offers a much better fit to the data than the $\Lambda \mathrm{CDM}$ curve (blue) [16]. Reproduced from [17].

large-scale clustering of galaxies [20]. The "Bullet" Cluster [21, 22], the aftermath of two colliding galaxy clusters, is also problematic for MOND [23].

\section{Dark Matter Condensate}

Much work has been done, e.g., [24, 25, 26, 27, 28], to reconcile the phenomenological success of CDM on cosmological scales with the empirical success of MOND on galactic scales. This talk summarizes an approach presented in a series of recent papers [29, 30, 31, 32]. The approach is a unified framework for the DM and MOND phenomena based on the rich and well-studied physics of superfluidity. The DM and MOND components have a common origin, representing different phases of a single underlying substance. The central idea is that DM forms a superfluid inside galaxies, with a coherence length of galactic size.

As is familiar from liquid helium, a superfluid at finite sub-critical temperature is best described phenomenologically as a mixture of two fluids [33, 34, 35]: $i$ ) the superfluid, which by definition has vanishing viscosity and carries no entropy; $i$ ) the "normal" component, comprised of massive particles, which is viscous and carries entropy. The fraction of particles in the condensate decreases with increasing temperature. Thus our framework naturally distinguishes between galaxies (where MOND is successful) and galaxy clusters (where MOND is not). Galaxy clusters have a higher velocity dispersion and correspondingly higher DM temperature. For $m \sim \mathrm{eV}$ we will find that galaxies are almost entirely condensed, whereas galaxy clusters are either in a mixed phase or entirely in the normal phase.

As a back-of-the-envelope calculation, we can estimate the condition for the onset of superfluidity ignoring interactions among DM particles. With this simplifying approximation, the requirement for superfluidity amounts to demanding that the de Broglie wavelength $\lambda_{\mathrm{dB}} \sim 1 / m v$ of DM particles should be larger than the interparticle separation $\ell \sim(m / \rho)^{1 / 3}$. This implies an upper bound on the particle mass, $m \lesssim\left(\rho / v^{3}\right)^{1 / 4}$. Substituting the value of $v$ and $\rho$ at virialization, given 
by standard collapse theory, this translates to [29, 30]

$$
m \lesssim 2.3\left(1+z_{\mathrm{vir}}\right)^{3 / 8}\left(\frac{M}{10^{12} h^{-1} M_{\odot}}\right)^{-1 / 4} \mathrm{eV},
$$

where $M$ and $z_{\mathrm{vir}}$ are the mass and virialization redshift of the object. Hence light objects form a Bose-Einstein condensate (BEC) while heavy objects do not.

Another requirement for Bose-Einstein condensation is that DM thermalize within galaxies. We assume that DM particles interact through contact repulsive interactions. Demanding that the interaction rate be larger than the galactic dynamical time places a lower bound on the interaction cross-section. For $M=10^{12} h^{-1} M_{\odot}$ and $z_{\mathrm{vir}}=2$, the result is $[29,30]$

$$
\frac{\sigma}{m} \gtrsim\left(\frac{m}{\mathrm{eV}}\right)^{4} \frac{\mathrm{cm}^{2}}{\mathrm{~g}}
$$

With $m \lesssim \mathrm{eV}$, this is just below the most recent constraint from galaxy cluster mergers [36], though such constraints should be carefully reanalyzed in the superfluid context.

Again ignoring interactions, the critical temperature for DM superfluidity is $T_{c} \sim \mathrm{mK}$, which intriguingly is comparable to known critical temperatures for cold atom gases, e.g. ${ }^{7} \mathrm{Li}$ atoms have $T_{c} \simeq 0.2 \mathrm{mK}$. Cold atoms might provide more than just a useful analogy — in many ways, our DM component behaves exactly like cold atoms. In cold atom experiments, atoms are trapped using magnetic fields; in our case, DM particles are attracted in galaxies by gravity.

\section{Superfluid Phase}

Instead of behaving as individual collisionless particles, the DM is more aptly described as collective excitations: phonons and massive quasi-particles. Phonons, in particular, play a key role by mediating a long-range force between ordinary matter particles. As a result, a test particle orbiting the galaxy is subject to two forces: the (Newtonian) gravitational force and the phononmediated force.

In the language of field theory, an (abelian) superfluid is described by the theory of a spontaneously broken global $U(1)$ symmetry, in a state of finite charge density. The relevant degree of freedom at low energy is the Goldstone boson for the broken symmetry, namely the phonon field $\theta$. The $U(1)$ symmetry acts non-linearly on $\theta$ as a shift symmetry, $\theta \rightarrow \theta+c$. In the non-relativistic regime and in the absence of external potentials, the theory should be Galilean invariant. According to the rules of effective field theory, we are instructed to write down all possible operators consistent with these symmetries. We will be interested in the case where there is a gravitational potential $\Phi$.

At leading order (LO) in the derivative expansion, the relevant building block is the (nonrelativistic) kinetic operator

$$
X=\dot{\theta}-m \Phi-\frac{(\vec{\nabla} \theta)^{2}}{2 m} .
$$

The most general LO action is an arbitrary function of this quantity [37, 38]:

$$
\mathscr{L}_{\mathrm{LO}}=P(X) .
$$


At finite chemical potential, $\theta=\mu t$, this action defines the grand canonical equation of state $P(\mu)$ of the superfluid. A straightforward calculation reveals that the energy density is

$$
\rho=m P_{, X}
$$

while the pressure is $P$. In other words, the type of superfluid is uniquely encoded in the choice of $P(X)$. Perturbations $\varphi=\theta-\mu t$ about this state describe phonon excitations.

In $[29,30]$ we conjectured that DM phonons are described by the non-relativistic MOND scalar action, ${ }^{2}$

$$
P(X)=\frac{2 \Lambda(2 m)^{3 / 2}}{3} X \sqrt{|X|}
$$

corresponding to $P \sim \mu^{3 / 2}$. Using the thermodynamic relation Eq. (3.3), this implies a polytropic equation of state $P \sim \rho^{3}$.

To mediate a MONDian force between ordinary matter, phonons must couple to baryons through

$$
\mathscr{L}_{\text {int }} \sim \frac{\Lambda}{M_{\mathrm{Pl}}} \theta \rho_{\mathrm{b}}
$$

which softly breaks the shift symmetry for $\theta .^{3}$ With this action, the phonon-mediated force and the usual Newtonian gravitational force together give an effective MOND force law, with the scale $\Lambda$ related to the critical acceleration via $\Lambda \sim \sqrt{a_{0} M_{\mathrm{Pl}}} \sim \mathrm{meV}$. Unlike "pure" MOND, however, the DM halo itself contributes to the Newtonian component of the acceleration. This contribution is negligible on distances probed by galactic rotation curves, but becomes comparable to the MOND component at distances of order the size of the superfluid core.

The superfluid interpretation has a number of advantages over other formulations of MOND. For starters it is more economical. There is no need to postulate additional degrees of freedom to modify gravity - the coherent phonon scalar field is enough. Secondly, the non-analytic nature of the kinetic term in Eq. (3.4) is more palatable, as it is intrinsically tied to the superfluid equation of state. The MONDian action given by Eq. (3.4) corresponds to $P \sim \rho^{3}$, as we will see shortly, which is analytic. In fact there is a well-known example of a theory with fractional power in cold atom systems - the Unitary Fermi Gas (UFG) [43, 44], describing fermionic atoms at unitary. The UFG superfluid action is fixed by non-relativistic scale invariance to the non-analytic form $\mathscr{L}_{\mathrm{UFG}}(X) \sim X^{5 / 2}[45]$.

\subsection{Properties of the Condensate and Phonons}

The form of the phonon action uniquely fixes the properties of the condensate through standard thermodynamics arguments. At finite chemical potential, $\theta=\mu t$, ignoring phonon excitations and

\footnotetext{
${ }^{2}$ The square-root form is necessary to ensure that the action is well-defined for time-like field profiles, and that the Hamiltonian is bounded from below [39].

${ }^{3}$ The baryon coupling in Eq. (3.5), while technically natural from an effective field theory point of view, picks out a preferred phase of the wavefunction. This seems unphysical. One possibility is that the coupling involves a difference of phase, which is a physical quantity, say between the local phase and the cosmological phase. Another possibility is that the shift symmetry is broken to a discrete subgroup through a $\cos \theta \rho_{\mathrm{b}}$ operator [40, 41, 42]. Expanding around the state at finite chemical potential $\theta=\mu t$, such a term would give Eq. (3.5) to leading order, albeit with an oscillatory prefactor.
} 


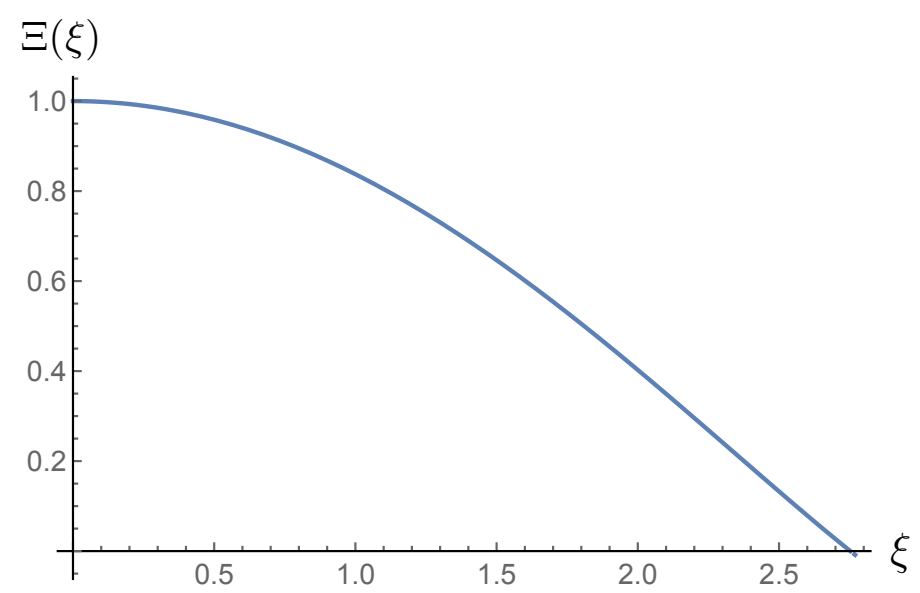

Figure 2: Numerical solution of Lane-Emden equation, Eq. (3.12).

gravitational potential to zero, the pressure of the condensate is given as usual by the Lagrangian density,

$$
P(\mu)=\frac{2 \Lambda}{3}(2 m \mu)^{3 / 2} .
$$

This is the grand canonical equation of state, $P=P(\mu)$, for the condensate. Differentiating with respect to $\mu$ yields the number density of condensed particles:

$$
n=\frac{\partial P}{\partial \mu}=\Lambda(2 m)^{3 / 2} \mu^{1 / 2} .
$$

Combining these expressions and using the non-relativistic relation $\rho=m n$, we find

$$
P=\frac{\rho^{3}}{12 \Lambda^{2} m^{6}} .
$$

This is a polytropic equation of state $P \sim \rho^{1+1 / n}$ with index $n=1 / 2$.

Including phonons excitations $\theta=\mu t+\phi$, the quadratic action for $\phi$ is

$$
\mathscr{L}_{\text {quad }}=\frac{\Lambda(2 m)^{3 / 2}}{4 \mu^{1 / 2}}\left(\dot{\phi}^{2}-\frac{2 \mu}{m}(\vec{\nabla} \phi)^{2}\right) .
$$

The sound speed can be immediately read off:

$$
c_{s}=\sqrt{\frac{2 \mu}{m}} .
$$

\subsection{Superfluid core profile}

Assuming hydrostatic equilibrium, we can compute the density profile of a spherically-symmetric DM superfluid core:

$$
\frac{1}{\rho(r)} \frac{\mathrm{d} P(r)}{\mathrm{d} r}=-\frac{4 \pi G_{\mathrm{N}}}{r^{2}} \int_{0}^{r} \mathrm{~d} r^{\prime} r^{\prime 2} \rho\left(r^{\prime}\right) .
$$


Substituting the equation of state given by Eq. (3.8), and introducing the dimensionless variables $\rho=\rho_{\text {core }} \Xi^{1 / 2}$ and $r=\sqrt{\frac{\rho_{\text {core }}}{32 \pi G_{\mathrm{N}} \Lambda^{2} m^{6}}} \xi$, with $\rho_{\text {core }}$ denoting the central density, Eq. (3.11) implies the Lane-Emden equation

$$
\left(\xi^{2} \Xi^{\prime}\right)^{\prime}=-\xi^{2} \Xi^{1 / 2}
$$

where ${ }^{\prime} \equiv \mathrm{d} / \mathrm{d} \xi$. The numerical solution, with boundary conditions $\Xi(0)=1$ and $\Xi^{\prime}(0)=0$, is shown in Fig. 2. The superfluid density profile is cored, not surprisingly, and therefore avoids the cusp problem of CDM.

The density is found to vanish at $\xi_{1} \simeq 2.75$, which defines the core size: $R_{\text {core }}=\sqrt{\frac{\rho_{\text {core }}}{32 \pi G_{\mathrm{N}} \Lambda^{2} m^{6}}} \xi_{1}$. Meanwhile the central density is related to the core mass as [46] $\rho_{\text {core }}=\frac{3 M_{\text {core }}}{4 \pi R_{\text {core }}^{3}} \frac{\xi_{1}}{\left|\Xi^{\prime}\left(\xi_{1}\right)\right|}$, with $\Xi^{\prime}\left(\xi_{1}\right) \simeq$ -0.5 . Combining these results, it is straightforward to solve for $\rho_{\text {core }}$ and $R$ :

$$
\begin{aligned}
& \rho_{\text {core }} \simeq\left(\frac{M_{\text {core }}}{10^{11} M_{\odot}}\right)^{2 / 5}\left(\frac{m}{\mathrm{eV}}\right)^{18 / 5}\left(\frac{\Lambda}{\mathrm{meV}}\right)^{6 / 5} 10^{-25} \mathrm{~g} / \mathrm{cm}^{3} \\
& R_{\text {core }} \simeq\left(\frac{M_{\text {core }}}{10^{11} M_{\odot}}\right)^{1 / 5}\left(\frac{m}{\mathrm{eV}}\right)^{-6 / 5}\left(\frac{\Lambda}{\mathrm{meV}}\right)^{-2 / 5} 28 \mathrm{kpc} .
\end{aligned}
$$

Remarkably, for $m \sim \mathrm{eV}$ and $\Lambda \sim \mathrm{meV}$ we obtain DM halos of realistic size! In the standard CDM picture a halo of mass $10^{12} M_{\odot}$ has a virial radius of $\sim 200 \mathrm{kpc}$. In our framework, the condensate radius can in principle be considerably smaller. For instance, with the fiducial values

$$
m=0.6 \mathrm{eV} ; \quad \Lambda=\mathrm{meV},
$$

we obtain a superfluid radius of $R_{\text {core }} \simeq 52 \mathrm{kpc}$ for a $M_{\text {core }}=10^{11} M_{\odot}$ core. This is beyond the largest distance observed with $\mathrm{HI}$ gas and ensures that we reproduce the success of MOND at fitting rotation curves [47]. The above calculation was at $T=0$, though realistically the DM is at finite, sub-critical temperature. A calculation is underway to include finite temperature corrections [48]. We expect the superfluid core to be surrounded by an atmosphere of DM particles in the normal phase following a nearly isothermal profile, akin to $[49,50]$.

\section{Phonon-Mediated MONDian Force}

Next we derive the phonon profile in galaxies, modeling the baryons as a static, sphericallysymmetric localized source for simplicity. We first focus on the zero-temperature analysis, where the Lagrangian is given by the sum of Eqs. (3.4) and (3.5). In the static spherically-symmetric approximation, $\theta=\mu t+\phi(r)$, the equation of motion reduces to

$$
\vec{\nabla} \cdot(\sqrt{2 m|X|} \vec{\nabla} \phi)=\frac{\alpha \rho_{\mathrm{b}}(r)}{2 M_{\mathrm{Pl}}}
$$

where $X(r)=\mu-m \Phi(r)-\frac{\phi^{\prime 2}(r)}{2 m}$. This can be readily integrated:

$$
\sqrt{2 m|X|} \phi^{\prime}=\frac{\alpha M_{\mathrm{b}}(r)}{8 \pi M_{\mathrm{P} 1} r^{2}} \equiv \kappa(r) .
$$


There are two branches of solutions, depending on the sign of $X$. We focus on the MOND branch (with $X<0$ ):

$$
\phi^{\prime}(r)=\sqrt{m}\left(\hat{\mu}+\sqrt{\hat{\mu}^{2}+\kappa^{2} / m^{2}}\right)^{1 / 2},
$$

where $\hat{\mu} \equiv \mu-m \Phi$. Indeed, for $\kappa / m \gg \hat{\mu}$ we have

$$
\phi^{\prime}(r) \simeq \sqrt{\kappa(r)} .
$$

In this limit the scalar acceleration on an ordinary matter particle is

$$
a_{\phi}(r)=\alpha \frac{\Lambda}{M_{\mathrm{Pl}}} \phi^{\prime} \simeq \sqrt{\frac{\alpha^{3} \Lambda^{2}}{M_{\mathrm{Pl}}} \frac{G_{\mathrm{N}} M_{\mathrm{b}}(r)}{r^{2}}} .
$$

To reproduce the MONDian result $a_{\mathrm{MOND}}=\sqrt{a_{0} \frac{G_{\mathrm{N}} M_{\mathrm{b}}(r)}{r^{2}}}$, we are therefore led to identify

$$
\alpha^{3 / 2} \Lambda=\sqrt{a_{0} M_{\mathrm{Pl}}} \simeq 0.8 \mathrm{meV} \Longrightarrow \alpha \simeq 0.86\left(\frac{\Lambda}{\mathrm{meV}}\right)^{-2 / 3}
$$

which fixes $\alpha$ in terms of $\Lambda$ through the critical acceleration.

As it stands, however, the $X<0$ solution is unstable. It leads to unphysical halos, with growing DM density profiles $[29,30]$. The instability can be seen by expanding Eq. (3.4) to quadratic order in phonon perturbations $\varphi=\phi-\bar{\phi}(r)$,

$$
\mathscr{L}_{\text {quad }}=\operatorname{sign}(\bar{X}) \frac{\Lambda(2 m)^{3 / 2}}{4 \sqrt{|\bar{X}|}}\left(\dot{\varphi}^{2}-2 \frac{\bar{\phi}^{\prime}}{m} \varphi^{\prime} \dot{\varphi}-2 \frac{\varphi^{\prime 2}}{m}\left(\bar{X}-\frac{\bar{\phi}^{\prime 2}}{2 m}\right)-\frac{2 \bar{X}}{m r^{2}}\left(\partial_{\Omega} \varphi\right)^{2}\right) .
$$

The kinetic term $\dot{\varphi}^{2}$ has the wrong sign for $\bar{X}<0$. (The $X>0$ branch, meanwhile, is stable but does not admit a MOND regime [29, 30].)

Since the DM condensate in actual galactic halos has non-zero temperature, however, we expect that the zero-temperature Lagrangian (Eq. (3.4)) to receive finite-temperature corrections in galaxies. At finite sub-critical temperature, the system is described phenomenologically by Landau's two-fluid model: an admixture of a superfluid component and a normal component. The finite-temperature effective Lagrangian is a function of three scalars [51]: $\mathscr{L}_{T \neq 0}=F(X, B, Y)$. The scalar $X$, already defined in Eq. (3.4), describes the phonon excitations. The remaining scalars are defined in terms of the three Lagrangian coordinates $\psi^{I}(\vec{x}, t), I=1,2,3$ of the normal fluid:

$$
\begin{aligned}
& B \equiv \sqrt{\operatorname{det} \partial_{\mu} \psi^{I} \partial^{\mu} \psi^{J}} ; \\
& Y \equiv u^{\mu}\left(\partial_{\mu} \theta+m \delta_{\mu}^{0}\right)-m \simeq \mu-m \Phi+\dot{\phi}+\vec{v} \cdot \vec{\nabla} \phi,
\end{aligned}
$$

where $u^{\mu}=\frac{1}{6 \sqrt{B}} \varepsilon^{\mu \alpha \beta \gamma} \varepsilon_{I J K} \partial_{\alpha} \psi^{I} \partial_{\beta} \psi^{J} \partial_{\gamma} \psi^{K}$ is the unit 4-velocity vector, and in the last step for $Y$ we have taken the non-relativistic limit $u^{\mu} \simeq(1-\Phi, \vec{v})$. By construction, these scalars respect the internal symmetries: i) $\psi^{I} \rightarrow \psi^{I}+c^{I}$ (translations); ii) $\psi^{I} \rightarrow R_{J}^{I} \psi^{J}$ (rotations); iii) $\psi^{I} \rightarrow \xi^{I}(\psi)$, with det $\frac{\partial \xi^{I}}{\partial \psi^{J}}=1$ (volume-preserving reparametrizations).

There is much freedom in specifying finite-temperature operators that stabilize the MOND profile. The simplest possibility is to supplement Eq. (3.4) with the two-derivative operator

$$
\Delta \mathscr{L}=M^{2} Y^{2}=M^{2}(\hat{\mu}+\dot{\phi})^{2},
$$


where we have specialized to the rest frame of the normal fluid, $\vec{v}=0$. This leaves the static profile given by Eq. (4.3) unchanged, but modifies the quadratic Lagrangian by $M^{2} \dot{\varphi}^{2}$, restoring stability for sufficiently large $M$. Specifically this is the case for

$$
M \gtrsim \frac{\Lambda m^{3 / 2}}{\sqrt{|\bar{X}|}} \sim 0.5\left(\frac{10^{11} M_{\odot}}{M_{\mathrm{b}}}\right)^{1 / 4}\left(\frac{\Lambda}{\mathrm{meV}}\right)^{1 / 2}\left(\frac{r}{10 \mathrm{kpc}}\right)^{1 / 2} m
$$

which, remarkably, is of order $\mathrm{eV}$ ! Hence, for quite natural values of $M$, this two-derivative operator can restore stability. Furthermore, this operator gives a contribution $\Delta P=M^{2} \mu^{2}$ to the condensate pressure, which obliterates the unwanted growth in the DM density profile. Instead, the pressure is positive far from the baryons, resulting in localized, finite-mass halos [29, 30].

\section{Observational Implications}

We conclude by listing some astrophysical implications of DM superfluidity.

Vortices: When spun faster than a critical velocity, a superfluid develops vortices. The typical angular velocity of halos is well above critical [29, 30], giving rise to an array of DM vortices permeating the disc $[52,53]$. It will be interesting to see whether these vortices can be detected through substructure lensing, e.g., with ALMA [54].

Galaxy mergers: A key difference with $\Lambda \mathrm{CDM}$ is the merger rate of galaxies. Applying Landau's criterion, we find two possible outcomes. If the infall velocity $v_{\text {inf }}$ is less than the phonon sound speed $c_{s}$, then halos will pass through each other with negligible dissipation, resulting in multiple encounters and a longer merger time. If $v_{\text {inf }} \gtrsim c_{\mathrm{S}}$, however, the encounter will excite DM particles out of the condensate, resulting in dynamical friction and rapid merger.

Reduced dynamical friction: More generally, the overall reduction in dynamical friction due to the superfluid nature of the DM halo alleviates a number of minor problems with CDM. Instead of being slowed down by dynamical friction, galactic bars in spiral galaxies should achieve a nearly constant velocity, as favored by observations [55]. Reduced dynamical friction would also help with the M81 group of galaxies [56] and could explain why the globular clusters around the Fornax dwarf spheroidal have not collapsed to its center [57, 58].

Dark-bright solitons: Galaxies in the process of merging should exhibit interference patterns (socalled dark-bright solitons) that have been observed in BECs counterflowing at super-critical velocities [59]. This can potentially offer an alternative mechanism to generate the spectacular shells seen around elliptical galaxies [60].

Globular clusters: Globular clusters are well-known to contain negligible amount of DM and are well-described by Newtonian gravity with baryons only. In cases where the external field effect is reliably small, globular clusters pose a problem for MOND, see e.g. [61]. In our case the presence of a significant DM component is necessary for MOND. If whatever mechanism responsible for DM removal in $\Lambda \mathrm{CDM}$ is also effective here, our model would predict DM-free (and hence MONDfree) globular clusters.

The elephant in the room is of course dark energy. Certainly it would be very compelling if cosmic acceleration could emerge from the same underlying substance as DM and MOND. Pre- 
liminary ideas along these lines will appear in a forthcoming publication [62].

Acknowledgments: I would like to warmly thank the organizers of the Dark Side of the Universe 2015 Workshop for a most enjoyable conference. I also thank Benjamin Elder, Alistair Hodson, Tom Lubensky, Vinicius Miranda, David Mota, Anushrut Sharma, Junpu Wang, Hans Winther, HongSheng Zhao, and particularly Lasha Berezhiani and Benoit Famaey for their collaboration and many stimulating conversations. This work is supported in part by NSF CAREER Award PHY-1145525, NASA ATP grant NNX11AI95G and a New Initiative Research Grant from the Charles E. Kaufman fund of The Pittsburgh Foundation.

\section{References}

[1] P. A. R. Ade et al. [Planck Collaboration], Astron. Astrophys. 571, A16 (2014) [arXiv:1303.5076 [astro-ph.CO]].

[2] S. S. McGaugh, J. M. Schombert, G. D. Bothun and W. J. G. de Blok, Astrophys. J. 533, L99 (2000) [astro-ph/0003001].

[3] S. S. McGaugh, Astrophys. J. 632, 859 (2005) [astro-ph/0506750].

[4] M. Vogelsberger et al., MNRAS 444, 1518 (2014) [arXiv:1405.2921 [astro-ph.CO]].

[5] K. A. Oman et al., Mon. Not. Roy. Astron. Soc. 452, no. 4, 3650 (2015) [arXiv:1504.01437 [astro-ph.GA]].

[6] M. Boylan-Kolchin, J. S. Bullock and M. Kaplinghat, MNRAS 415, L40 (2011) [arXiv:1103.0007 [astro-ph.CO]].

[7] M. S. Pawlowski, J. Pflamm-Altenburg and P. Kroupa, MNRAS 423, 1109 (2012) [arXiv:1204.5176 [astro-ph.GA]].

[8] R. A. Ibata et al., Nature 493, 62 (2013) [arXiv:1301.0446 [astro-ph.CO]].

[9] R. A. Ibata et al., Astrophys. J. 784, L6 (2014) [arXiv:1403.2389 [astro-ph.GA]].

[10] H. Zhao, B. Famaey, F. L§ghausen and P. Kroupa, Astron. Astrophys. 557, L3 (2013) [arXiv:1306.6628 [astro-ph.GA]].

[11] M. Milgrom, Astrophys. J. 270, 365 (1983).

[12] R. H. Sanders and S. S. McGaugh, Ann. Rev. Astron. Astrophys. 40, 263 (2002) [astro-ph/0204521].

[13] B. Famaey and S. McGaugh, Living Rev. Rel. 15, 10 (2012) [arXiv:1112.3960 [astro-ph.CO]].

[14] A. H. Broeils, Astron. and Astrophys. 256,19 (1992).

[15] K. G. Begeman, A. H. Broeils and R. H. Sanders, MNRAS 249, 523 (1991).

[16] J. A. Sellwood and S. S. McGaugh, Astrophys. J. 634, 70 (2005).

[17] M. Milgrom, arXiv:0908.3842 [astro-ph.CO].

[18] A. Aguirre, J. Schaye and E. Quataert, Astrophys. J. 561, 550 (2001) [astro-ph/0105184].

[19] J. D. Bekenstein, Phys. Rev. D 70, 083509 (2004) [Erratum-ibid. D 71, 069901 (2005)] [astro-ph/0403694]. 
[20] C. Skordis, D. F. Mota, P. G. Ferreira and C. Boehm, Phys. Rev. Lett. 96, 011301 (2006) [astro-ph/0505519].

[21] D. Clowe, A. Gonzalez and M. Markevitch, Astrophys. J. 604, 596 (2004) [astro-ph/0312273].

[22] D. Clowe et al., Astrophys. J. 648, L109 (2006) [astro-ph/0608407].

[23] G. W. Angus, B. Famaey and H. Zhao, MNRAS 371, 138 (2006) [astro-ph/0606216].

[24] L. Blanchet, Class. Quant. Grav. 24, 3529 (2007) [astro-ph/0605637].

[25] J. -P. Bruneton, S. Liberati, L. Sindoni and B. Famaey, JCAP 0903, 021 (2009) [arXiv:0811.3143 [astro-ph]].

[26] B. Li and H. Zhao, Phys. Rev. D 80, 064007 (2009) [arXiv:0904.2935 [astro-ph.CO]].

[27] C. M. Ho, D. Minic and Y. J. Ng, Phys. Lett. B 693, 567 (2010) [arXiv:1005.3537 [hep-th]].

[28] J. Khoury, Phys. Rev. D 91, 024022 (2015) [arXiv:1409.0012 [hep-th]].

[29] L. Berezhiani and J. Khoury, Phys. Lett. B 753, 639 (2016) [arXiv:1506.07877 [astro-ph.CO]].

[30] L. Berezhiani and J. Khoury, Phys. Rev. D 92, 103510 (2015) [arXiv:1507.01019 [astro-ph.CO]].

[31] J. Khoury, arXiv:1507.03013 [astro-ph.CO].

[32] J. Khoury, to appear in Phys. Rev. D., arXiv:1602.05961 [astro-ph.CO]

[33] L. Tisza, C. R. Acad. Sci. 207, 1035 (1938); 207, 1186 (1938).

[34] F. London, Phys. Rev. 54, 947 (1938).

[35] L. D. Landau, J. Phys. (USSR) 5, 71 (1941); 11, 91 (1947).

[36] D. Harvey et al., Science 347, no. 6229, 1462 (2015) [arXiv:1503.07675 [astro-ph.CO]].

[37] M. Greiter, F. Wilczek and E. Witten, Mod. Phys. Lett. B 3, 903 (1989).

[38] D. T. Son, hep-ph/0204199.

[39] J. -P. Bruneton and G. Esposito-Farese, Phys. Rev. D 76, 124012 (2007) [Erratum-ibid. D 76, 129902 (2007)] [arXiv:0705.4043 [gr-qc]].

[40] J. Villain, J. Phys. (France) 36, 581 (1975).

[41] J. V. José, L. P. Kadanoff, S. Fitzpatrick and D. R. Nelson, Phys. Rev. B 16, 1217 (1977).

[42] R. D. Kamien, HUTP-89/A025.

[43] W. Zwerger, ed. The BCS-BEC Crossover and the Unitary Fermi Gas, Lecture Notes in Physics, Vol. 836 (Springer- Verlag, Berlin Heidelberg, 2012).

[44] S. Giorgini, L. P. Pitaevskii and S. Stringari, Rev. Mod. Phys. 80, 1215 (2008) [arXiv:0706.3360 [cond-mat.other]].

[45] D. T. Son and M. Wingate, Annals Phys. 321, 197 (2006) [cond-mat/0509786].

[46] S. Chandrasekhar, An introduction to the study of stellar structure, Dover Publications, New York (1957).

[47] B. Famaey and J. Khoury, to appear.

[48] J. Khoury, T. Lubensky, A. Miranda and A. Sharma, to appear. 
[49] Z. Slepian and J. Goodman, Mon. Not. Roy. Astron. Soc. 427, 839 (2012) [arXiv:1109.3844 [astro-ph.CO]].

[50] T. Harko and E. J. M. Madarassy, JCAP 1201, 020 (2012) [arXiv:1110.2829 [astro-ph.GA]].

[51] A. Nicolis, arXiv:1108.2513 [hep-th].

[52] M. P. Silverman and R. L. Mallett, Gen. Rel. Grav. 34, 633 (2002).

[53] T. Rindler-Daller and P. R. Shapiro, Mon. Not. Roy. Astron. Soc. 422, 135 (2012) [arXiv:1106.1256 [astro-ph]].

[54] Y. Hezavehvet al., Astrophys. J. 767, 9 (2013) [arXiv:1210.4562 [astro-ph.CO]].

[55] V. P. Debattista and J. A. Sellwood, Astrophys. J. 493, L5 (1998) [astro-ph/9710039].

[56] P. Kroupa, arXiv:1409.6302 [astro-ph.GA].

[57] S. D. Tremaine, J. P. Ostriker and L. Spitzer Jr., Astrophys. J. 196, 407 (1975).

[58] S. D. Tremaine, Astrophys. J. 203, 345 (1976).

[59] C. Hamner, J. J. Chang, P. Engels and M. A. Hoefer, Phys. Rev. Lett. 106, 065302 (2011).

[60] A. P. Cooper et al., Astrophys. J. Lett. 743, L21 (2011).

[61] R. Ibata et al., Astrophys. J. 738, 186 (2011) [arXiv:1106.4909 [astro-ph.CO]].

[62] L. Berezhiani, J. Khoury and J. Wang, to appear. 